\title{
Informal allopathic provider knowledge and practice regarding control and prevention of TB in rural Bangladesh
}

\author{
Qazi S. Islam ${ }^{a, *}$, Syed M. Ahmed ${ }^{a, b}$, Mohammad A. Islam $^{c}$, Anita S. Chowdhury ${ }^{a}$, Bodrun N. Siddiquea ${ }^{c}$ \\ and Mohammad A. Husain ${ }^{\text {d }}$
}

\begin{abstract}
${ }^{a}$ BRAC Research and Evaluation Division, Dhaka 1212, Bangladesh; ${ }^{b}$ Centre of Excellence for Universal Health Coverage, James P. Grant School of Public Health/BRAC University, Dhaka 1212, Bangladesh; 'BRAC Health Nutrition and Population Programme, Dhaka 1212,

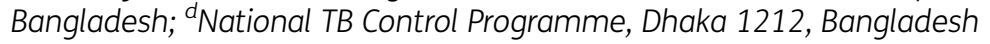

*Corresponding author: Present address: Bangladeshi-Canadian Community Services, 2899 Danforth Avenue, Toronto, Ontario, Canada M4C 1M3; Tel: +1 416357 8548; Fax: +1 416698 8127; E-mail: tarunnnn@yahoo.com

Received 26 July 2013; revised 2 April 2014; accepted 7 April 2014

\begin{abstract}
Background: BRAC (formerly Bangladesh Rural Advancement Committee), in collaboration with the National Tuberculosis Control Programme, provides one full-day training on TB to make informal allopathic providers knowledgeable for managing TB in rural Bangladesh. This study explored the knowledge and practices of the providers receiving the above training in the control and prevention of TB.
\end{abstract}

\begin{abstract}
Methods: The study was conducted in 30 subdistricts, with 30 trained and 30 untrained providers randomly selected from each subdistrict. Approximately 3\% (49/1800) did not provide complete information. Pre-tested structured and semi-structured questionnaires were used.

Results: TB was commonly perceived as a disease of only males $(66.1 \%, 1157 / 1751)$. Only one-quarter knew about the bacterial cause of TB. Very few providers $(2.1 \%, 36)$ had adequate knowledge regarding prevention of TB. They also lacked knowledge about TB treatment duration $(71.6 \%, 1253)$, the meaning of DOTS (directly observed treatment, short course) $(26.0 \%, 455)$ and multidrug resistance $(20.6 \%, 360)$. Antibiotics $(79.7 \%$, $1396)$ and cough syrup $(75.0 \%, 1313)$ were commonly prescribed by providers despite symptoms suggestive of TB. However, $70.2 \%$ (613) and $74.5 \%$ (650) of trained providers' knowledge and practice scores were equal to or more than the mean scores ( $\geq 6.97$ and $\geq 6.6$, respectively), whereas they were only $49.5 \%(435)$ and $64.2 \%(563)$, respectively, among untrained providers ( $p<0.0001)$.
\end{abstract}

Conclusions: Misperception, lack of knowledge and irrational use of antibiotics are challenges that need to be addressed for controlling and preventing TB efficiently.

Keywords: Bangladesh, BRAC, DOTS, Informal healthcare providers, TB

\section{Introduction}

A large private sector is actively engaged in health service delivery in Bangladesh. In rural areas, unqualified and unregistered informal private providers (traditional healers and allopathic providers such as village doctors and drug shop attendants) are the dominant healthcare providers. ${ }^{1}$ They provide $60 \%$ of healthcare services in rural areas in the informal sector. ${ }^{2}$ Informal healthcare providers are deeply embedded in the local community and culture, with easy accessibility, and provide inexpensive services to villagers. They manage patients mostly with drugs and advice, sometimes with advice only. These unqualified practitioners rarely give advice for laboratory investigation, but irrational use of prescription of antibiotics by them is very common. ${ }^{3}$
TB is a major public health problem in Bangladesh, which is sixth among the 22 high-burden TB countries in the world. ${ }^{4}$ However, Bangladesh has been successful in controlling TB, and the National Tuberculosis Control Programme (NTP) has achieved its targets in case detection (70\%) and treatment success rate $(85 \%))^{5}$ However, the TB control programme still has some challenges to overcome to sustain the current momentum. A significant number of patients having a cough for $\geq 3$ weeks in rural areas sought care from informal allopathic providers, but the majority of providers do not notify the patients or refer them to appropriate places for case detection owing to their lack of knowledge on TB, its diagnosis and treatment. ${ }^{6}$ These inappropriate practices pose a great threat to the efforts of controlling TB efficiently.

(C) The Author 2014. Published by Oxford University Press on behalf of Royal Society of Tropical Medicine and Hygiene. All rights reserved. For permissions, please e-mail: journals.permissions@oup.com. 
The NTP in partnership with non-governmental organisations (NGOs) implements the TB control strategy across the country. The NTP of the Government of Bangladesh launched the DOTS (directly observed treatment, short course) strategy in $1993 .{ }^{5}$ The Bangladesh NTP has recently shown considerable interest in exploring various options to involve the private sector, including informal providers, in controlling TB. BRAC (one of the largest NGOs) in collaboration with the NTP started a 1-day orientation training (one of the activities of the TB control programme) for village doctors, drug shop attendants, medical professionals and NGO workers in BRAC's TB control programme areas since 2005 to enable providers to play an informed role in preventing and controlling TB. Through this orientation training, the programme aims to create awareness among providers about the symptoms, transmission and prevention of TB. Consequently, providers are expected to refer presumptive TB patients to appropriate places with proper amenities and expertise. So far, no independent evaluation has been conducted to see how far the objectives of the training have been translated into practice. Thus, this study focused on exploring the current knowledge and practice of the trained and untrained (no training on TB) informal healthcare providers with respect to TB.

\section{Materials and methods}

\section{Study setting}

BRAC, a non-governmental development organisation, in collaboration with the NTP of the Government of Bangladesh is currently implementing a community-based TB control programme in 297 subdistricts in 42 districts of Bangladesh. ${ }^{5}$ The BRAC TB control programme actually started in 1984 as a pilot project in one subdistrict and was extended to 10 other subdistricts in 1992. BRAC was the first NGO to sign a Memorandum of Understanding with the Government of Bangladesh in 1994 to expand DOTS services. Community health workers of BRAC provide door-to-door TB care in rural areas. ${ }^{8}$ They identify patients who have had a cough for $\geq 3$ weeks and refer these presumptive TB patients to a sputum testing laboratory for TB detection. In addition, BRAC is involved in giving 1-day training on TB to informal providers at the subdistrict level under the guidelines of the NTP by the formal providers. It is an ongoing process and has yet to cover all informal allopathic providers in each subdistrict. These informal providers (village doctors and drug shop attendants) mainly consult patients at their work places/chambers in local markets. All providers for this study were selected from local market places in 30 subdistricts (receiving and not receiving training).

\section{Study population and sample size}

Trained informal allopathic providers were found in local markets using the participation lists (in the orientation programme) obtained from the local BRAC office. Untrained providers were selected through informal discussion with the trained providers from the same markets. From the 42 districts of the BRAC TB control programme areas, 30 subdistricts were selected using a probability proportional to size strategy, i.e. more subdistricts

Table 1. Sociodemographic characteristics of healthcare providers

\begin{tabular}{|c|c|c|c|c|}
\hline Characteristic & Trained $n=873(\%)$ & Untrained $n=878(\%)$ & All n=1751 (\%) & p-value \\
\hline Mean (SD) age (years) & $40.7(11.2)$ & $37.2(11.4)$ & $38.9(11.4)$ & $<0.001$ \\
\hline \multicolumn{5}{|l|}{ School education } \\
\hline Secondary (6-10 years) & $334(38.3)$ & $325(37.0)$ & $659(37.6)$ & NS \\
\hline Mean (SD) working hours/day & $10.7(2.4)$ & $10.7(2.6)$ & $10.7(2.5$ & NS \\
\hline Mean (SD) working years & $15.6(10.4)$ & $11.9(9.8)$ & $13.9(10.3)$ & $<0.001$ \\
\hline \multicolumn{5}{|l|}{ Monthly income (\%) } \\
\hline BDT $<2500$ & $16(1.8)$ & $19(2.2)$ & $35(2.0)$ & \\
\hline BDT 2500-5000 & $116(13.3)$ & $133(15.1)$ & $249(14.2)$ & NS \\
\hline \multicolumn{5}{|c|}{ Self-rated poverty status of households in last year } \\
\hline Deficit (\%) & $118(13.5)$ & $125(14.2)$ & $243(13.9)$ & \multirow[t]{2}{*}{ NS } \\
\hline No deficit (\%) & $755(86.5)$ & $753(85.8)$ & $1508(86.1)$ & \\
\hline
\end{tabular}


were selected from bigger districts. The sample size for informal healthcare providers was determined on the basis of maximum proportioning (0.5) with a $5 \%$ significance level and a $5 \%$ precision. The minimum calculated sample size for each category of informal healthcare providers was approximately 400. To capture the variations in the 30 subdistricts, the sample size was increased to $900(400 \times 2.25)$ considering design effect 2.25 . Thus, the study proposed to include 900 trained and 900 untrained providers. In total, 60 informal allopathic providers (30 trained and 30 untrained) were selected from each subdistrict. A total of 873 trained and 878 untrained providers participated.

\section{Data collection}

Structured and semi-structured questionnaires were used to collect information from the providers through face-to-face interviews. The questionnaires were pre-tested outside the study areas to ascertain consistency, appropriateness of language and sequencing of questions and to gain an insight into the field operation procedures needed. The final questionnaires were modified and updated on the basis of field testing. Adept interviewers were recruited to take information from the respondents. A 5-day intensive training course was organised for the interviewers. A total of 1800 informal allopathic healthcare providers were invited for participation. Among them, 873 trained and 878 untrained participated. All interviews were held at the chambers/ work places or drug shops/pharmacies. The duration of each interview was 30-45 min. Effective and supportive supervision was ensured on a continual basis at all levels of the study.

\section{Data processing and analysis}

All available data were edited, coded and cleaned using SPSS Statistics for Windows v.16.0 (SPSS Inc., Chicago, IL, USA) for analysis. The sample mean was computed for continuous variables such as age, years of schooling, years of professional experiences, and knowledge and practice scores. Age groups were constructed on the basis of the mean. TB knowledge and reported standard practice towards TB were determined by a score based on the answers given to questions. One point was given for each correct answer for constructing knowledge scores. Similarly, one point was given for each practice related to TB management. A respondent was rated as having adequate knowledge if he/she gave more correct answers than the mean correct answers for all the

Table 2. Proportion of respondents giving correct answer to each knowledge question

\begin{tabular}{|c|c|c|c|c|}
\hline \multirow[t]{2}{*}{ Knowledge variable } & \multicolumn{3}{|l|}{ Provider type } & \multirow[t]{2}{*}{$p$-value } \\
\hline & Trained $n=873(\%)$ & Untrained $n=878(\%)$ & All $n=1751$ (\%) & \\
\hline TB is a disease both of males and female [1] & $239(27.4)$ & $237(27.0)$ & $476(27.2)$ & NS \\
\hline Main cause of TB is a bacterium [2] & $260(29.8)$ & $189(21.5)$ & $449(25.6)$ & $<0.001$ \\
\hline $\begin{array}{l}\text { Main suggestive symptom of TB is persistent } \\
\text { cough [3] }\end{array}$ & $838(96.0)$ & $842(95.9)$ & $1680(95.9)$ & NS \\
\hline $\begin{array}{l}\text { Main transmission route of TB is sneezing/ } \\
\text { coughing (airborne) [4] }\end{array}$ & $700(80.2)$ & $646(73.6)$ & 1346 (76.9) & $<0.01$ \\
\hline $\begin{array}{l}\text { Prevention of adult pulmonary TB: early detection } \\
\text { and starts TB treatment without delay }\end{array}$ & $20(2.3)$ & $16(1.8)$ & $36(2.0)$ & NS \\
\hline $\begin{array}{l}\text { Had knowledge about the relationship between } \\
\text { TB and malnutrition [5] }\end{array}$ & $432(49.5)$ & $375(42.8)$ & $807(46.1)$ & $<0.001$ \\
\hline $\begin{array}{l}\text { Had knowledge about the duration of cough that } \\
\text { might indicate TB ( } \geq 3 \text { weeks) [6] }\end{array}$ & $712(81.6)$ & $671(76.4)$ & $1383(79.0)$ & $<0.004$ \\
\hline $\begin{array}{l}\text { Had knowledge about BRAC facility/laboratory for } \\
\text { sputum testing [7] }\end{array}$ & $837(95.9)$ & $714(81.3)$ & $1551(88.6)$ & $<0.001$ \\
\hline $\begin{array}{l}\text { Had knowledge about times of testing sputum } \\
\text { of individual for detecting TB bacterium } \\
\text { (three times) [8] }\end{array}$ & $426(48.8)$ & $359(40.9)$ & $785(44.8)$ & $<0.01$ \\
\hline Had knowledge about free treatment policy [9] & $857(98.2)$ & $825(94.0)$ & $1682(96.1)$ & $<0.001$ \\
\hline $\begin{array}{l}\text { Had knowledge about standard TB treatment } \\
\text { duration ( } 6 \text { months) [10] }\end{array}$ & $681(78.0)$ & $572(65.2)$ & $1253(71.6)$ & $<0.01$ \\
\hline Had knowledge about the meaning of DOTS [11] & $333(38.1)$ & $123(13.9)$ & $455(26.0)$ & $<0.001$ \\
\hline Had knowledge about multidrug resistance [12] & $205(23.5)$ & $155(17.7)$ & $360(20.6)$ & $<0.004$ \\
\hline Mean (SD) knowledge score [1-12] & $7.5(1.7)$ & $6.5(1.8)$ & $6.97(1.8)$ & $<0.001$ \\
\hline Proportion with knowledge score $\geq 6.97$ & $613(70.2)$ & $435(49.5)$ & 1048 (59.9) & $<0.001$ \\
\hline
\end{tabular}

NS: not significant; DOTS: directly observed treatment, short course.

Square brackets indicate the serial number of knowledge variables for constructing knowledge score. 
respondents. Furthermore, a respondent was rated as having adequate practice if he/she gave more correct answers than the mean correct answers related to TB management for all the respondents. A $\chi^{2}$ test and independent t-test were calculated to assess statistical significance. Multiple regression analysis was performed to capture the significant association demonstrated.

\section{Results}

The sociodemographic profile of the study sample is shown in Table 1. Informal allopathic providers who received training were older (mean age 41 years vs 37 years) and more experienced (mean working years 16 vs 12 years) than their counterparts. Both trained and untrained providers had around 12 years of schooling (mean) and had a mean monthly income of 11000 12000 Bangladeshi Taka (US\$140-155). The majority of providers $(86.1 \%, 1508)$ self-rated their household poverty status as having no deficit. Around 3\% (49) of the selected providers did not participate in the study.

Less than one-third of providers $(27.2 \%, 476)$ were aware of TB as a disease of males and females (Table 2). TB was commonly perceived as a disease of only males $(66.1 \%, 1157)$ (data not shown). Knowledge on the bacterial cause of TB was inadequate among trained $(29.8 \%, 260)$ and untrained providers $(21.5 \%, 189)$ $(p<0.001)$. Moreover, factors such as tobacco smoking and filthy environments were known as causes of TB by $75.0 \%$ (1313) and 53.0\% (928) of respondents, respectively (data not shown). The main suggestive symptom of TB (chronic cough) was well known both to trained and untrained providers (95.9\%,1680). Knowledge about the main transmission route of TB (sneezing/coughing) was equally conspicuous among trained $(80.2 \%, 700)$ and untrained providers $(73.6 \%, 646 ; p<0.01)$. Simultaneously, trained and untrained providers did not show adequate knowledge on early detection and treatment start with no delay $(2.0 \%, 36)$ as the best strategy for prevention of adult TB (Table 2). They mainly focused on refraining from smoking/quitting $(60.0 \%, 1051)$ and maintaining a healthy environment $(48.0 \%, 840)$ as the prime strategy for prevention of TB (data not shown). Knowledge about the relationship between TB and nutrition was low ( $49.5 \%$ vs $42.8 \%, 432$ vs 375$)$ for trained and untrained providers, respectively; $p<0.001)$. Knowledge regarding a duration of cough for $\geq 3$ weeks being the main suggestive symptom of TB was found to be adequate among trained providers $(81.6 \%, 712)$ compared with untrained providers $(76.4 \%$, $671 ; p<0.004$ ) (Table 2). Furthermore, more comprehensive knowledge of trained providers was observed in the case of knowledge about the BRAC laboratory for sputum testing $(95.9 \%$ vs $81.3 \% ; 837$ vs 714; $p<0.001)$, the free TB treatment policy (98.2\% vs $94 \% ; 857$ vs $825 ; p<0.001)$ and the standard duration of TB treatment ( 6 months) (78\% vs $65.2 \%$; 681 vs $512 ; p<0.01$ ). However, knowledge regarding the meaning of DOTS (i.e. taking medicine in front of health workers) was found to be inadequate ( $38.1 \%$ vs $13.9 \%$; 333 vs $123 ; p<0.001)$. Knowledge about multidrug resistance was also found to be very low $(20.6 \%, 360)$.

Table 3. Proportion of respondents giving answers to each practice question

\begin{tabular}{|c|c|c|c|c|}
\hline Practice variable & Trained $n=873(\%)$ & Untrained $n=878(\%)$ & All $n=1751(\%)$ & p-value \\
\hline Mean TB patients seen in last month [n (SD)] & $4.7(9.6)$ & $4.5(10.37)$ & $4.5(9.9)$ & NS \\
\hline Looked for other associated TB symptoms when suspecting TB [1] & $867(99.3)$ & $859(97.8)$ & $1726(98.6)$ & $<0.02$ \\
\hline Prescribed sputum test when suspecting TB [2] & $851(97.5)$ & $836(95.2)$ & $1687(96.3)$ & $<0.02$ \\
\hline Prescribed antibiotics when suspecting TB & $698(80.0)$ & $698(79.5)$ & $1396(79.7)$ & NS \\
\hline $\begin{array}{l}\text { Referred TB suspects to appropriate places for } \\
\text { further management [3] }\end{array}$ & $869(99.6)$ & $871(99.2)$ & $1740(99.4)$ & NS \\
\hline \multicolumn{5}{|l|}{ Most usual places } \\
\hline BRAC & $812(93.0)$ & 666 (75.9.) & $1478(84.4)$ & $<0.001$ \\
\hline Advised about nutritious food and nutrition management [7] & $841(96.3)$ & $799(91.0)$ & $1640(93.7)$ & $<0.001$ \\
\hline Mean (SD) practice score [1-7] & $6.7(0.60)$ & $6.4(0.84)$ & $6.6(0.73)$ & $<0.001$ \\
\hline Proportion with practice score $\geq 6.6$ & $650(74.5)$ & $563(64.2)$ & $1213(69.3)$ & $<0.002$ \\
\hline
\end{tabular}


Table 4. Mean TB knowledge ${ }^{a}$ and practice ${ }^{b}$ score by sociodemographic characteristics $(n=1751)$

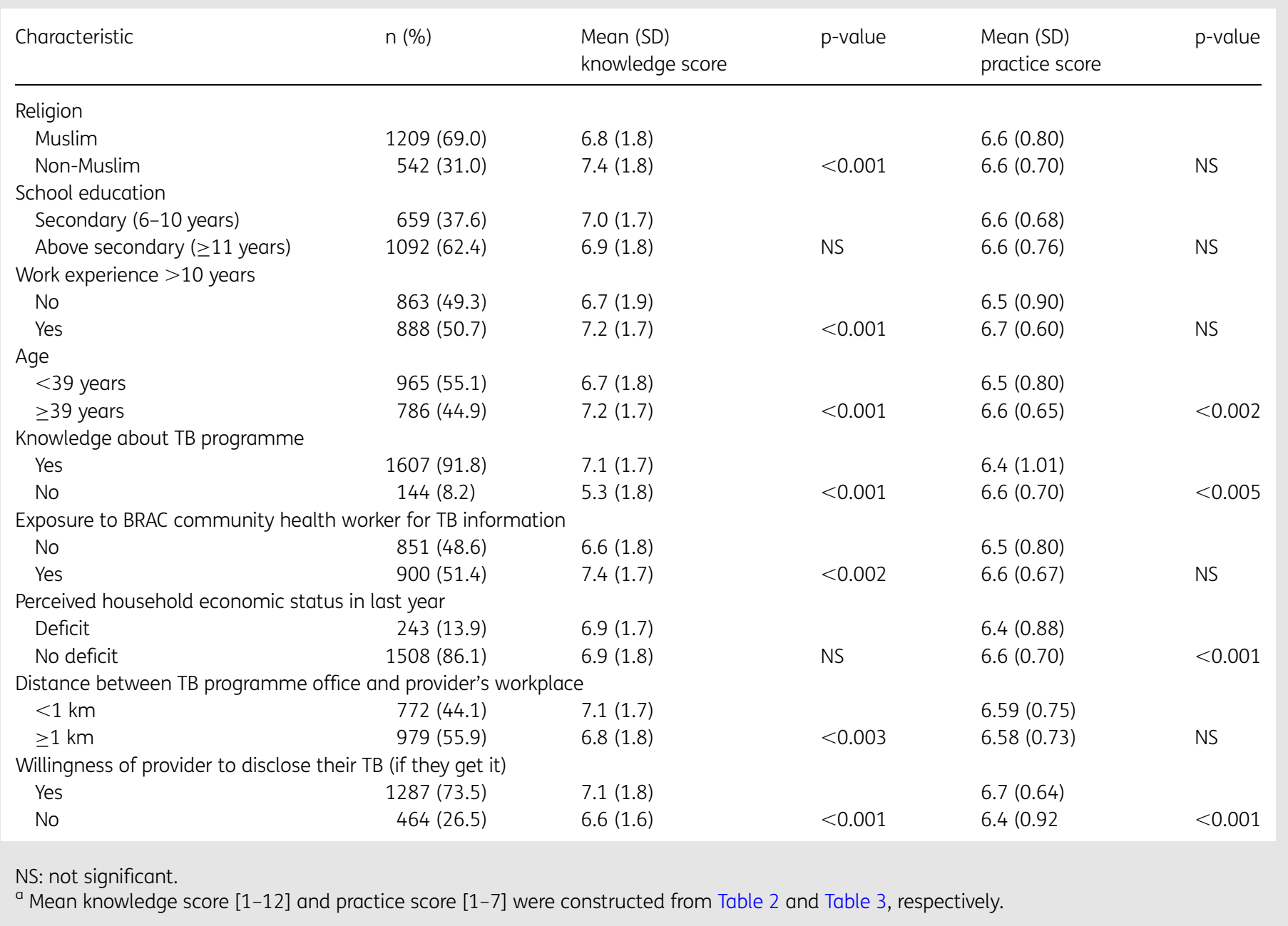

Each provider consulted around 16 presumptive TB patients and five TB patients on average in the last month (Table 3). Both trained and untrained providers reported that they usually (97.8-99.3\%, 859-867) looked for other associated symptoms related to TB when suspecting its presence. Advice for sputum testing $(96.3 \%, 1687)$ and for referring presumptive TB cases elsewhere was universal $(99.4 \%, 1740)$. The most common place of referral was BRAC by trained providers $(93 \%, 812 ; p<0.001)$ and the government hospital by untrained providers (75.9\%, 667; $\mathrm{p}<0.004$ ). However, a great majority of presumptive TB patients (79-87\%, 694-759) were treated with medicines on first consultation by trained and untrained providers. Antibiotics (79.7\%, 1396) and/or cough syrup (74-76\%, 646-667) were commonly prescribed. Besides, trained providers advised on adverse effects of TB drugs, against spitting sputum indiscriminately, and taking food of appropriate quality and quantity in greater proportion than untrained providers.

Both the mean (7.5) knowledge score of the trained providers and the proportion $(70.2 \%, 613)$ of these with a knowledge score higher than the mean were higher than the population average $(6.97$ and $59.9 \%, 1048$ respectively; $p<0.001$ ) (Table 2 ). Furthermore, the mean (6.7) practice score of the trained providers and the proportion $(74.5 \%, 650)$ of these with a practice score higher than the mean were higher than the population average (6.6 and 69.3\%, 1213 respectively; $p<0.002$ ) (Table 3). The higher knowledge and practice scores were observed by informal providers who knew about the TB programme and had work experience of $>10$ years (Table 4 ). The results of multiple regression analysis (Table 5) shows that informal providers who had training showed a significant association with increased knowledge and practice scores. Further investigation reveals that as the informal providers became more aware about the TB programme, progressed in age and had regular exposure to BRAC community health workers, their knowledge scores increased significantly $(p<0.05)$.

\section{Discussion}

A large proportion of presumptive TB cases in Bangladesh often go to rural informal allopathic providers ${ }^{6}$ despite the availability 


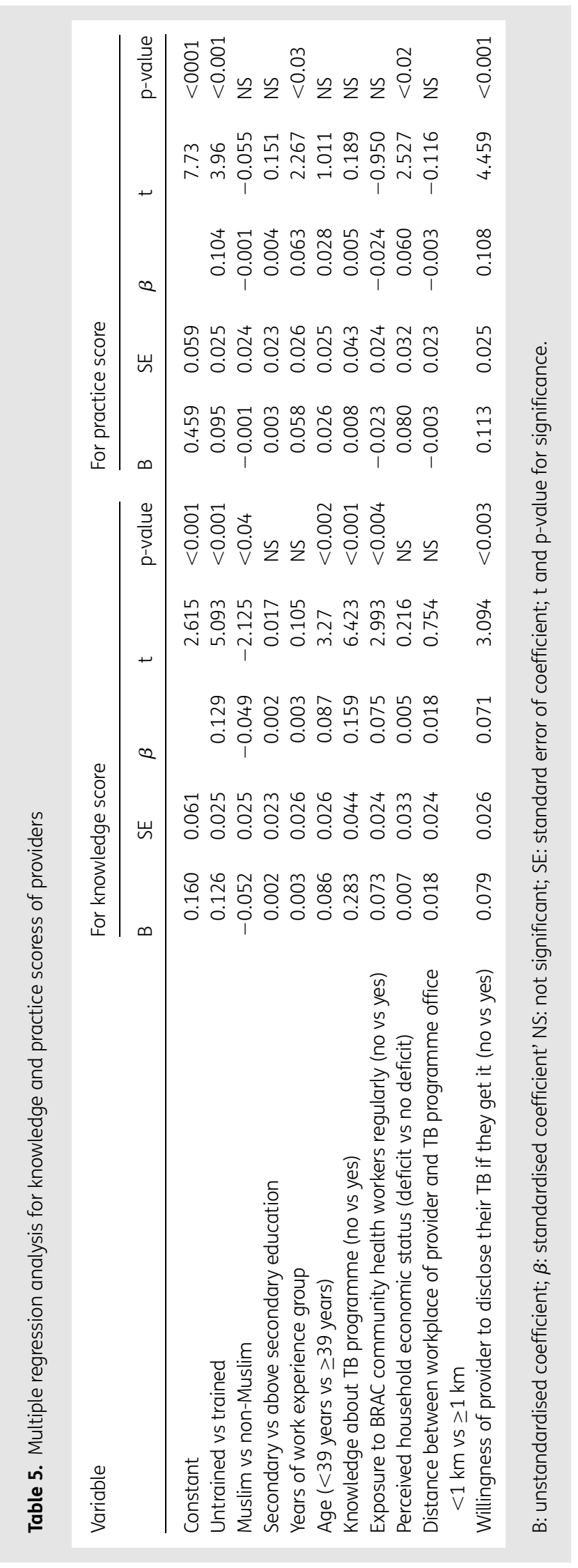

of formal allopathic providers or formal healthcare facilities such as public hospitals. Thus, the TB programme in a given area cannot reach the goal of universal coverage without the involvement of informal healthcare providers. ${ }^{9,10}$ The NTP of Bangladesh has already taken initiative to involve informal allopathic providers in the TB control programme under a public-private mix approach. The NTP started a 1-day training programme all over the country that began in $2005^{11}$ to increase awareness among informal allopathic providers regarding the importance of TB control and of referring patients to appropriate places. This study aimed to understand how effective this orientation programme has been in improving the management of presumptive TB cases in the community. The findings reveal that trained providers still had misperceptions (i.e. TB is a disease of only males) as well as a lack of knowledge on the bacterial cause of $\mathrm{TB}$, the best strategy for prevention of adult TB, the standard duration of TB treatment, the meaning of DOTS and multidrug resistance. Also, irrational use of antibiotics for relieving the cough symptom was very common among informal healthcare providers. The programmatic implications of these findings are discussed below.

It is interesting to observe that TB was still perceived as a disease of males after so many years of TB programme implementation. Whatever the underlying reasons, such misperception could interfere with the proper diagnosis of presumptive TB cases by rural informal allopathic providers. For example, in Afghanistan, TB is perceived as a disease of only females ${ }^{12}$ and the detection rate of TB among females is higher in Afghanistan. ${ }^{13}$ Another widely held perception among providers was that TB was primarily caused by smoking, similar to that among communities in Ethiopia and Tanzania. ${ }^{14,15}$ The practical implication of this is that providers would not give due emphasis to non-smokers when suspecting TB cases.

Bangladesh adopted the DOTS strategy in 1993 for TB control and this currently covers the whole nation. ${ }^{5}$ DOTS is important to ensure patient adherence to treatment until completion. Previously, knowledge of DOTS was totally absent among these providers. ${ }^{6}$ However, findings from this study show that the situation has started to change for the better. This is commendable as lack of appropriate knowledge on DOTS was observed even among qualified practitioners in Kenya and Nigeria. ${ }^{16,17}$

Knowledge of providers on suggestive symptom of TB (chronic cough for $\geq 3$ weeks) and transmission of TB was highly comprehensive, better than that observed for qualified practitioners in Pakistan. ${ }^{18}$ This will ultimately help informal allopathic providers to detect suspected cases early and to send them for sputum testing. These providers were found to be familiar with the national policies on TB, such as free-of-cost treatment for TB patients. Similar findings were observed in China and India. ${ }^{19,20}$ This knowledge improvement can be attributed to the orientation training, besides the role of mass media and BRAC's health workers in disseminating priority information in BRAC's TB control programme areas.

Although there was an improvement in initiating sputum testing for presumptive TB cases and appropriate referral from previous years, ${ }^{6}$ irrational/improper use of antibiotics remains a very common practice and a matter of concern. Several reasons may be responsible: usual practice by rural providers for many years; lack of knowledge that inappropriate use of antibiotics is harmful; and pressure from patients for immediate relief. Such use of antibiotics by providers should be abandoned to prevent antibiotic resistance and to reduce the delay in TB diagnosis. If there is a delay in initiating diagnosis and treatment, there is a 
probability of spreading the causative bacteria from the source. On the other hand, advice by providers to patients on continuing the full course of TB medicine and adverse reactions to TB drugs, as observed in this study, is welcome as this will help to prevent the emergence of multidrug resistance. Even the providers' advice on nutrition can be tapped for future nutritional management programme for TB patients.

\section{Conclusions}

It can be said that the 1-day orientation training imparted by BRAC in collaboration with the NTP of the Government of Bangladesh has had some positive impact on improving the knowledge and practices of informal allopathic providers towards TB management. However, such short training is inadequate in allaying misperceptions and increasing the depth of knowledge and should be addressed by expanded training with a scaled-up curriculum. In training, irrational use of antibiotics should be discouraged and attempts should be made to disseminate proper drug information to informal healthcare providers.

The main limitation of this study is that the responses of the providers were not validated by observations of their practice pattern. Another limitation of this study is that the randomness in recruiting the respondents of the training was not guaranteed. Therefore, it is possible that the 'trained providers' had better knowledge and practice or had favourable background characteristics prior to the training, thus biasing the comparison with the 'untrained' favouring the 'trained'.

Authors' contributions: QSI, SMA and MAI conceived the study; QSI, SMA, MAI, ASC and MAH designed the study protocol; QSI, ASC and BNS implemented the study; QSI analysed the data and drafted the manuscript; QSI, SMA and MAI critically revised the manuscript for intellectual content. All authors read and approved the final manuscript. QSI is guarantor of the paper.

Acknowledgements: The authors thank the staff of BRAC for their help with data collection. The authors are also grateful to the study respondents who shared their time and experience with them.

Funding: This study was funded by the Global Fund to Fight AIDS, Tuberculosis and Malaria (GFATM) through the BRAC TB programme.

Competing interests: None declared.

Ethical approval: This study was reviewed and approved by the Research and Evaluation Division of BRAC. All respondents were informed that their responses would remain anonymous, and verbal consent was obtained before interviewing respondents.

\section{References}

1 Ahmed SM, Hossain MA, Chowdhury MR. Informal sector providers in Bangladesh: how equipped are they to provide rational health care? Health Policy Plan 2009;24:467-78.

2 Cockcroft A, Anderson N, Milne D et al. What did the public think of the health services reform in Bangladesh? Three national communitybased surveys 1999-2003. Health Res Policy Syst 2007;5:1-7.

3 Ahmed SM, Rashid SF, Ghafur T et al. Healthcare providers and service provision. In: Chowdhury AMR, Iqbal K (editors). The State of Health in
Bangladesh 2007. Health Workforce in Bangladesh: Who Constitutes the Healthcare System? Dhaka, Bangladesh: James P. Grant School of Public Health, BRAC University, 2008;7-12.

4 WHO. Global Tuberculosis Report 2012. Geneva, Switzerland: World Health Organization; 2012. WHO?HTM?TB/2012.6.

5 National Tuberculosis Control Programme. Tuberculosis Control in Bangladesh. Annual Report 2012. Dhaka, Bangladesh: National Tuberculosis Control Programme, Director General of Health Services.

6 Islam QS, Karim F, Sulaiman M et al. Role of rural private healthcare providers in detecting and treating tuberculosis cases. In: Socioeconomic Divides in Tuberculosis Control. Research Monograph Series No. 41. Dhaka, Bangladesh: BRAC Research and Evaluation Division, 2009;42-59.

7 Rangan SG, Juvekar SK, Monrankar SN et al. Tuberculosis control in rural India: lessons from public-private collaboration. Int J Tuberc Lung Dis 2004;8:552-9.

8 Bangladesh Tuberculosis Control Programme, NGO component. Annual Report 2012. Dhaka, Bangladesh: BRAC Health Programme.

9 Dewan PK, Lal SS, Lonnroth $\mathrm{K}$ et al. Improving tuberculosis control through public-private collaboration in India: literature review. BMJ 2006;332:574-8.

10 Agarwal SP, Sehgal S, Lal SS. Public-private mix in the Revised National TB Control programme. In: Agwarwall SP, Cahuhan LS (editors). Tuberculosis Control in India. Delhi, India: Elsevier, 2005;135-44.

11 Islam S, Harries AD, Malhotra S et al. Training of community healthcare providers and TB case detection in Bangladesh. Int Health 2013;5: 223-7.

12 Islam QS, Ahmed SM, Rahmen Tet al. Knowledge and attitude of rural population towards TB and perception of barriers in accessing to TB services in two provinces of Afghanistan. BRAC Research and Evaluation Division. http://www.bracresearch.org/reports_details.php? scat $=8 \&$ tid $=516 \& \mathrm{~V}=$ Accessed on 12.06.2013 [accessed 12 April 2014].

13 Sabawoon W, Sato H. Sex difference in tuberculosis in Afghanistan: a national cohort study. Mycobacterial Dis 2012;2:1-5. doi:10.4172/ 2161-1068.1000115.

14 Mesfin M, Tasew T, Tareke I et al. Community knowledge, attitudes, and practices on pulmonary tuberculosis and their choices of treatment supervisor in Tigray, Northern Ethiopia. Ethiop J Health Dev 2005;19: 21-7.

15 Mangesho PE, Shayo E, Makunde WH et al. Community knowledge, attitudes and practices towards tuberculosis and its treatment in Mpwapwa district, central Tanzania. Tanzan Health Res Bull 2007;9: 38-43.

16 Ayaya SO, Sitienel J, Orero W et al. Knowledge, attitudes and practices of private practitioners on tuberculosis among HIV/AIDS patients in Eldoret, Kenya. East Afr Med J 2003;80:83-90.

17 Dosumu EA. Survey of knowledge, attitudes, and practices regarding tuberculosis among general and private medical practitioners in Nigeria. Afr J Respir Med 2008;17-9.

18 Ahmed M, Fatmi Z, Ali S, Ahmed J et al. Knowledge, attitude and practice of private practitioners regarding TB-DOTS in a rural district of Sindh, Pakistan. J Ayub Med Coll Abbottabad 2009;21:28-31.

19 Gai R, Xu L, Wang X et al. The role of village doctors on tuberculosis control and the DOTS strategy in Shandong Province, China. Biosci Trends 2008;2:181-6.

20 Banerjee A, Sharma BV, Ray A et al. Acceptability of traditional healers as directly observed treatment providers in tuberculosis control in a tribal area of Andhra Pradesh. India. Int J Tuberc Lung Dis 2004;8: 1260-5. 